UCRL-JC-119583

PREPRINT

\title{
Mechanical Design of Recirculating Accelerator Experiments for Heavy-Ion Fusion
}

\author{
V. Karpenko
}

This paper was prepared for submittal to the 1995 Particle Accelerator Conference and International Conference on High-Energy Accelerators

Dallas, TX

May 1-5, 1995

May 23, 1995

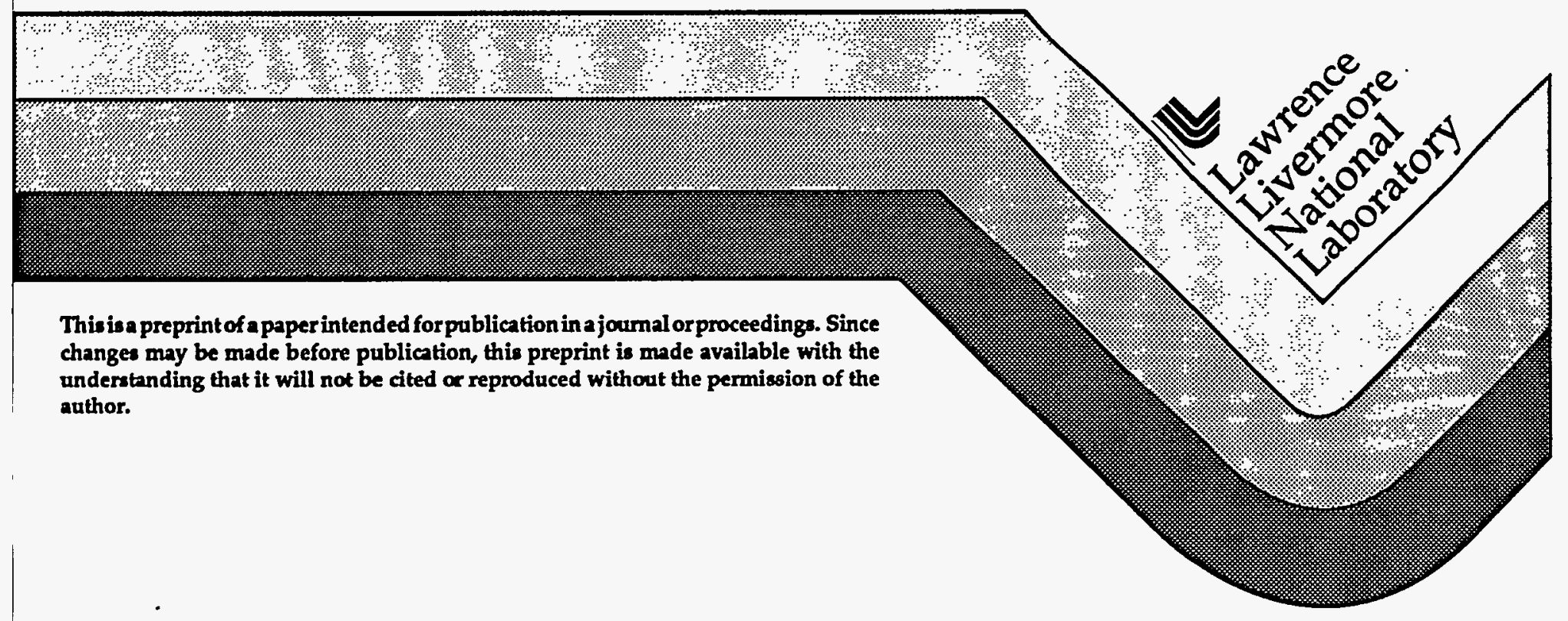




\section{DISCLAIMER}

This document was prepared as an account of work sponsored by an agency of the United States Government. Neither the United States Government nor the University of California nor any of their employees, makes any warranty, express or implied, or assumes any legal liability or responsibility for the accuracy; completeness, or usefulness of any information, apparatus, product, or process disclosed, or represents that its use would not infringe privately owned rights. Reference herein to any specific commercial product, process, or service by trade name, trademark, manufacturer, or otherwise, does not necessarily constitute or imply its endorsement, recommendation, or favoring by the United States Government or the University of California. The views and opinions of authors expressed herein do not necessarily state or reflect those of the United States Government or the University of California, and shall not be used for advertising or product endorsement purposes. 


\section{DISCLAIMER}

Portions of this document may be illegible in electronic image products. Images are produced from the best available original document. 


\title{
MECHANICAL DESIGN OF RECIRCULATING ACCELERATOR EXPERIMENTS FOR HEAVY-ION FUSION
}

\author{
Victor Karpenko \\ Lawrence Livermore National Laboratory, P.O. Box 808, L-481, Livermore, CA 94550
}

\section{INTRODUCTION}

Recirculating induction accelerators have been studied as a potential low cost driver for inertial fusion energy. At LLNL, we are developing a small (4.5-m diameter), scaled, experimental machine which will demonstrate many of the engineering solutions of a full scale driver.[1] The small recirculator will accelerate singly ionized potassium ions from 80 to $320 \mathrm{keV}$ and 2 to $8 \mathrm{~mA}$, using electric dipoles for bending and permanent magnet quadrupoles for focusing in a compact periodic lattice. While very compact, and low cost, this design alllows the investigation of most of the critical physics issues associated with space-charge-dominated beams in future IFE power plant drivers. Figure 1. illustrates the overall physics design and components which make up the design of the recirculator. Additionally, a straight magnetic-transport experiment is being carried out to test diagnostics and magnetic transport in preparation for the recirculator.

\section{DESCRIPTION OF THE RECIRCULATOR}

This small recirculator will have an aperture of 68 $\mathrm{mm}$ for the focusing element, and a half-lattice period of $360 \mathrm{~mm}$. The full recirculator will consist of 40 halflattice periods. Figure 2 illustrates a $90^{\circ}$ section of the ring. The electric dipoles and quadrupoles and associated stand-off distances occupy the majority of the axial space of the half-lattice period, thereby, dictating its mechanical design (see Fig. 3). The present experimental plans call for accelerating the $\mathrm{K}^{+}$ions through 15 laps. To minimize beam losses during the 15 laps, a vacuum of beter than $10^{-}$ 8 torr is required. Each half-lattice period is individually assembled, aligned, and installed on the ring using a coordinate measuring machine and multiple optical surveying transits to meet the demanding $250 \mu \mathrm{m}$ positioning tolerance of the dipoles and quadrupoles. The half-lattice periods are mounted on kinematic mounts to facilitate removal for operational reasons without the need for realignment.

\section{MECHANICAL DESIGN}

The recirculator half lattice period is constructed from two non-magnetic stainless steel subassemblies each of which house the electric dipole and permanent magnet quadrupoles. Space constraints of the electric dipole, quadrupole, induction core, and the ring geometry required dipole vacuum housing to have a trapezoidal shape (see Fig. 4). Investigation into manufacturing techniques revealed that the most cost effective method is to machine six individual plates and assemble them by either welding or vacuum brazing. The dipole is mounted on the trapezoidal top flange. Each of the dipoles is supported by a vacuum compatible machinable ceramic. This ceramic mount is in turn mounted to a commercially available optic mount which allows precision roll and pitch adjustments of the plates. The optical mount is mounted to allow two additional translations of the dipole assembly. Two $40 \mathrm{kV}$ feedthroughs and a $32 \mathrm{~mm}$ diagnostic port are provided on the dipoles mounting flange. The quadrupole subassembly consists of a precision tube, three $32 \mathrm{~mm}$ diagnostic ports, flanges, and a welded bellows. The key design feature is the precision machining of the outside diameter of the tube to locate the quadrupole magnet. The quadrupole magnet is clamped to this surface during assembly. This feature establishes the datum for aligning each half-lattice period to the plane of the ring. The half-lattice period is joined with an insulated bolted assembly capable of holding off 2 $\mathrm{kV}$. Each of the subassemblies requires heat treating to remove residual magnetism resulting from the welding procedures.

The vacuum connection on the lower trapezoidal flange also serves as the mounting structure for the halflattice period. A large circular flange at the interface between the ion pump and the bottom spool piece provides the structure for the kinematic mount.

\section{VACUUM DESIGN}

The vacuum design requirement for the recirculator of an operating pressure of less than $10^{-8}$ torr requires the use of metal seals and ultra high vacuum design features. The design of the half lattice period utilizes a number of metal seal designs. Where applicable, conventional copper sealed knife edge and metal o-ring seals are used. All components will be cleaned and handled following standard UHV practices. Each half lattice period will have a $60 \mathrm{l} / \mathrm{s}$ ion pump.

The sealing of the two trapezoid flanges to the dipole body required an unique application of existing metal seal technology. After reviewing a number of metal seal designs for ease of fabrication, reliability and cost, a version of the knife edge joint was selected. This seal design was adapted by MDC specifically for rectangular flanges. Its application to the trapezoidal shape of the dipole body was natural adaptation. The unique feature of this design is the orientation of the knife edge. Unlike the conventional design, the tapered face of the knife edge points to the interior of the flange. This design features-

\section{琵零

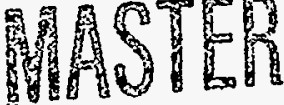 \\ MHes}

DISTRIBUTION OF THIS DO 
allows the sealing surface to be machined on a conventional milling machine with a specially ground tool An additional feature of this process is that the machine marks are essentially circumferential, similar to lathe machining, therefore additional polishing is not requiring .

The accelerating gap in the half-lattice period consists of a $127 \mu \mathrm{m}$ sheet of Kapton sandwiched between two stainless flanges. A single metal o-ring provides the vacuum seal.

\section{ALIGNMENT PROCESS PROCEDURE}

The alignment for the recirculator was derived from techniques used for the precision alignment of SLAC and other similar accelerators. The alignment method consists of using multiple optical surveying transits referenced against at least two benchmarks to absolutely position the individual half-lattice periods into the recirculator beam path diameter. The method reduces the chance of propagating errors during assembly and provides the capability for repeatable realignment after performing regular maintenance, upgrades or disassembly.

The alignment method is comprised of four processes. The first process is the alignment of the beam tube assembly that includes the aligning of the electric dipole and the magnet using a coordinate measuring machine. The second process is alignment of the recirculator platform relative to a bench mark using precision, optical transits and triangulation. The third process is the mounting and connecting of the beam tube assembly to the recirculator platform, and the final process is the alignment of the beam tubes relative to the plane of the ring.

A critical dimension for the recirculator is the relationship of the dipole center line with the quadrupole axis for each half-lattice. This dimension will be adjusted and measured before installation using a Coordinate Measuring Machine that will project the quadrupole axis by sweeping out the inner and outer diameter of the beam tube. The next step is to sweep the surface of the electric dipoles and project its center line. The dipole adjustment stage will be used to manipulate the dipole centerline relative to the quadrupole axis. A final check will be made to define the locations of the beam tube, dipole, and the tooling balls mounted on the half-lattice structure. These tooling balls will act as reference targets for the optical alignment during assembly of the recirculator.

\section{MAGNETIC TRANSPORT EXPERIMENT}

As a precursor to the development and deployment of the recirculator, it is necessary to study the magnetic transport of intense ion beams. The Magnetic Transport Experiment (MTE) is primarily intended to provide an experimental apparatus to assess the role of electrons in magnetic beam transport. It will also provide a test-bed for the development of intercepting and non-intercepting beam diagnostics and permit characterization of the potassium ion beam before injection into the ring. The MTE consists of three spools, each supporting two recirculator-design magnetic quadrupoles (see Fig. 5). Each spool provides a $35 \mathrm{~mm}$ radius beam-tube and access ports to allow insertion of beam diagnostics, and vacuum pumping ports. An entry spool consisting of a beam tube, a single magnetic quadrupole, and a gate valve provides connection to the potassium ion source and matching section. The MTE terminates in a diagnostics module designed to house a Faraday cup detector and a developmental optical diagnostic consisting of a scintillator and imaging system. The MTE is approximately $2.5-\mathrm{m}$ in length, excluding the terminal diagnostics box.

Spool diagnostics for each stage include LBLdesigned slit scanners for measuring beam emittance and an LLNL-developed four-lobe capacitive probe. Access ports are designed to permit insertion of the capacitive probe without disassembly of the spool, which permits modifications to be made with minimal impact. Vacuum pumping is achieved by a 60 liter/second ion pump on each spool, with appropriate provisions for attachment of roughing pumps as required. Vacuum requirements are less stringent than the recirculator since multiple passes of the beam will not occur. Pressures in the range of $1 \times 10-6$ torr are adequate for this experiment. Pressure will be measured by monitoring ion pump current. The gate valve at the entry stage allows the MTE to be brought up to air separately from the source and matching section, which saves pump-down time and reduces the likelihood of contaminating these stages.

Alignment of the MTE is accomplished by using optical bore-sighting with precision cross-hair targets inserted in the beam tube. Initial alignment will establish the beam line by referencing back to the source diode. Later stages will be added using the established line and referencing back to the source only if problems occur. Alignment tolerances for the beam line quadrupoles are $250 \mu \mathrm{m}$.

The MTE, which is scheduled to be operational in May 1995, will offer a convenient apparatus with which to explore fundamental physics issues associated with recirculating ion accelerators as well as to develop the unique diagnostics required for multiple lap operation. In addition, it is proving to be a valuable exercise for resolving fabrication, assembly, alignment, and operational issues prior to ring deployment.

\section{REFERENCES}

[1] A. Friedman et. al., these Proceedings.

\section{ACKNOWLEDGMENTS}

This work was performed under the auspices of the U.S. Department of Energy by Lawrence Livermore National Laboratory under contract No. W-7405-Eng-48. 


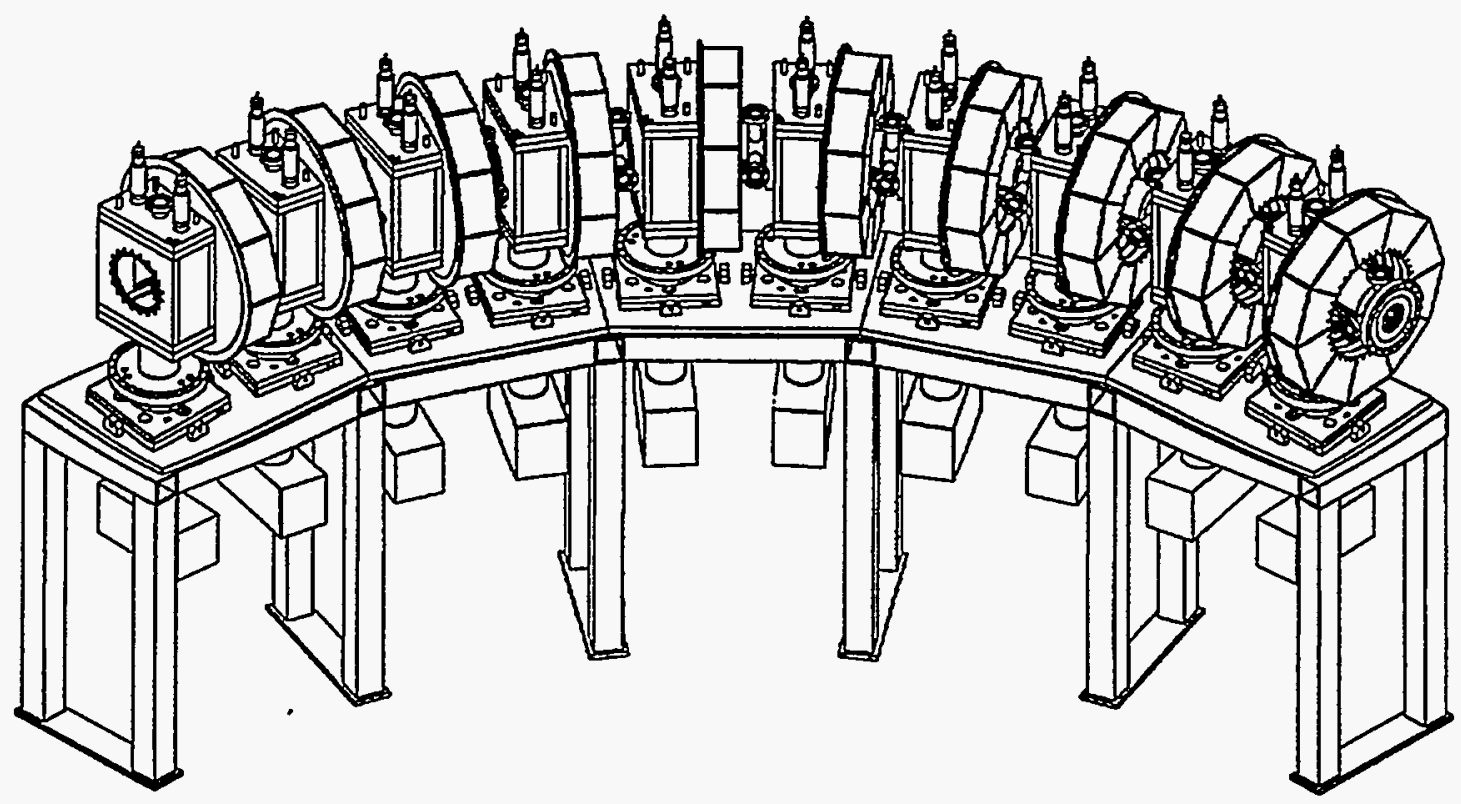

40-00-0595-1168 pbo1

Figure 1. A $90^{\circ}$ segment of the recirculator. 


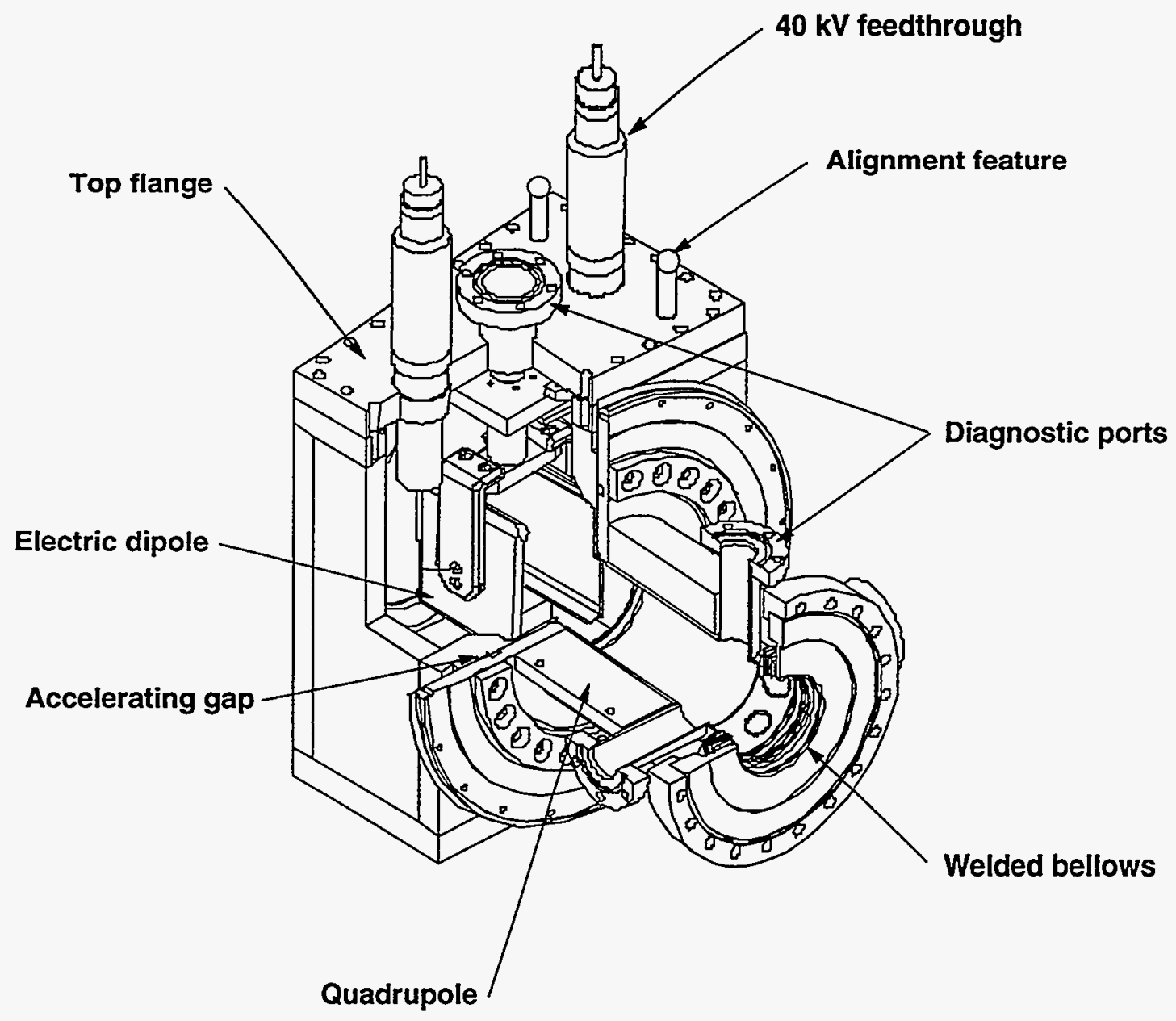

40-00-0595-1169 pb01

Figure 2. A quarter section view of the half-lattice period illustrating its major features. 


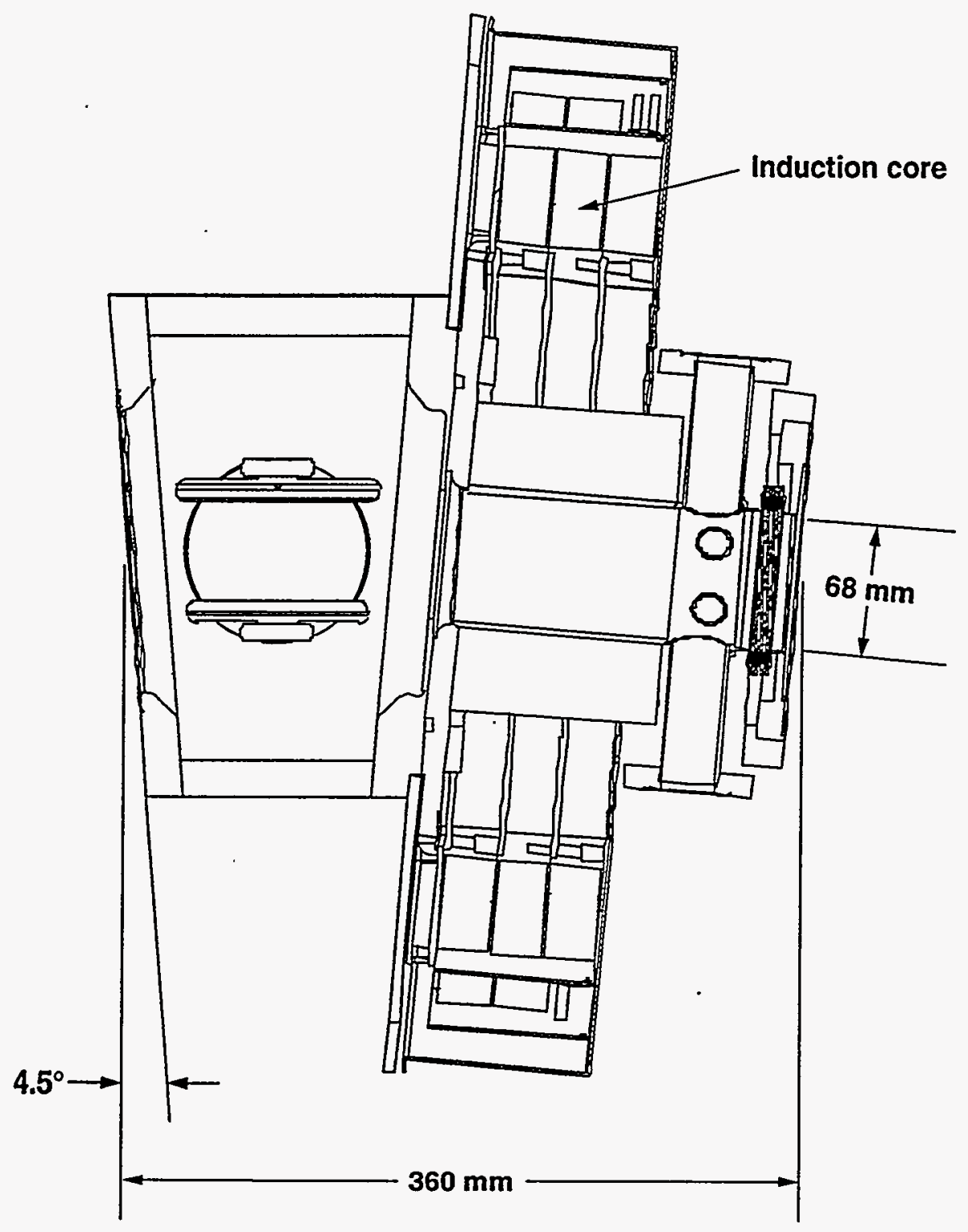

40-00-0595-1170 pb01

Figure 3. A half section view of the half-lattice period illustrating the integration of the compact design 


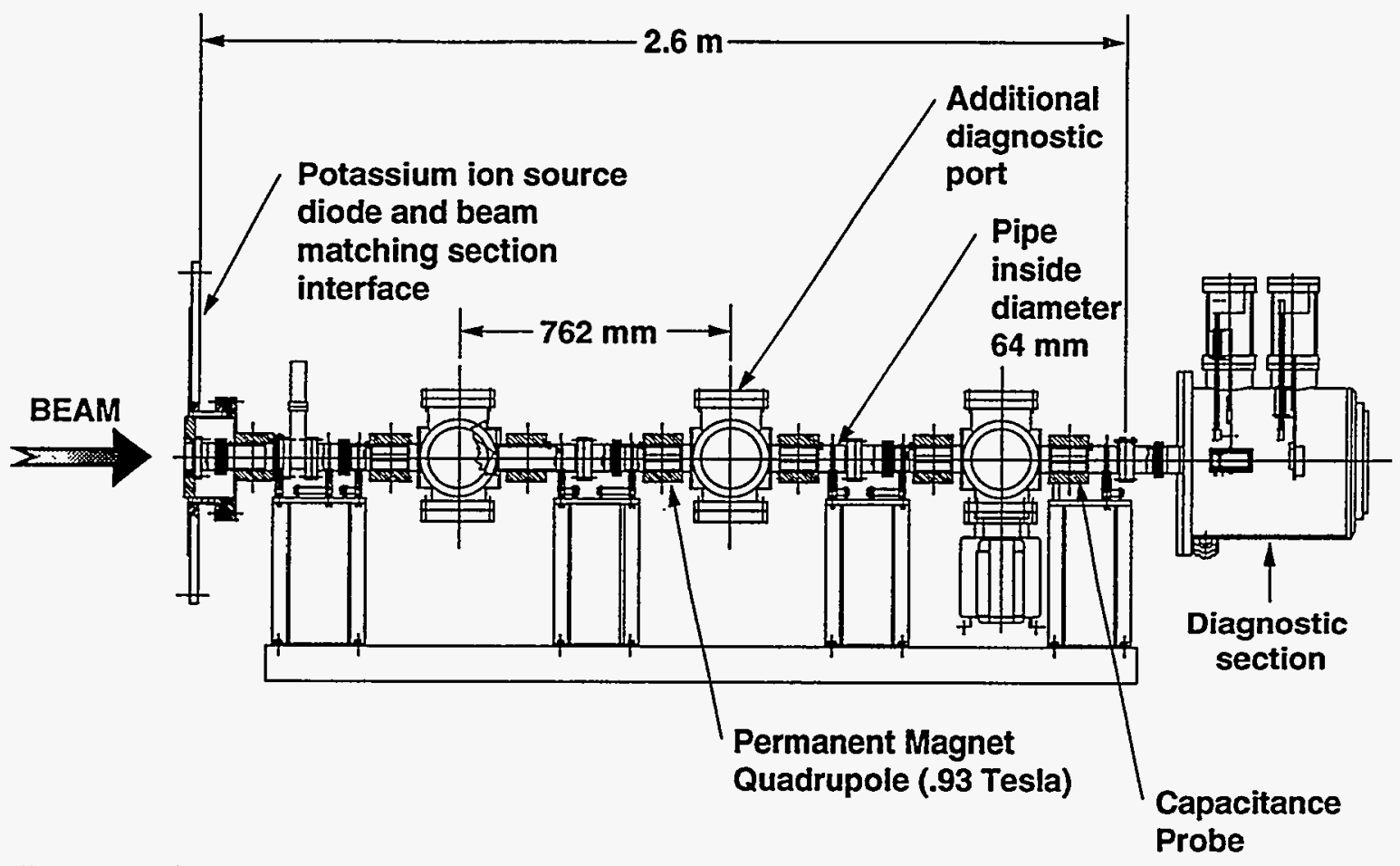

40-00-0595-1171 pbot

Figure 4. Linear configuration of the Magnetic Transport Experiment 